\title{
Dagmar Bunk: Bericht über den Stand der Konversion
}

Die Konversion im Allgemeinen (nicht zu verwechseln mit Rekon - in diesem Projekt wurden die Bestände von 1500 bis 1850 im SWB erfasst) startete im Sommer 1993 mit der Konversion des Lesesaalbestandes.

Bei allen Arten der Konversion wird anhand des alphabetischen Kataloges konvertiert, weil nur er vollständig ist. In den Standort- oder systematischen Katalogen sind dagegen die Folgebände bei mehrbändigen Werken nicht immer nachgewiesen.

\section{Lesesaalkonversion}

Mehrere Stammkräfte der Titelaufnahme und verschiedene Hilfskräfte, darunter auch Studentinnen der (damals noch) Fachhochschule für Bibliothekswesen, arbeiteten stundenweise daran. Die Lesesaal-Konversion konnte im Mai 1997 abgeschlossen werden.

\section{Handschriftenlesesaal}

Danach wurde der Alphabetische Katalog des Handschriftenlesesaals in Angriff genommen; er ist seit Mitte 1998 fertig konvertiert.

\section{Unterstützung durch die Zukunfts- offensive Baden-Württemberg}

Ab 1998 wurden vom Land zusätzliche Mittel zur Verfügung gestellt, mit denen die Erfassung des „neueren Altbestandes" per EDV-Katalog vorangetrieben werden sollte. Das Projekt ist vorläufig auf vier Jahre begrenzt, läuft also noch bis Ende 2001. Die Leitung des Projektes liegt bei der Autorin des Beitrags.

\section{Alphabetischer Hauptkatalog}

Um in der vorgesehenen Zeit einen definierten Abschnitt des Alphabetischen Hauptkataloges fertig stellen zu können, wurde die Konversion der Er- scheinungsjahre 1980 - 1988 beschlossen. Ziel ist der unmittelbare Anschluss an den Bestand im OPAC. Nach Schätzungen des Leiters der Katalogabteilung ist mit etwa 450000 Lokalsätzen zu rechnen, die in der vierjährigen Laufzeit bei $4 \frac{1}{2}$ ständig besetzten Stellen zur Bearbeitung anstehen.

Jeder Kasten aus dem Alphabetischen Katalog wird nach den zu konvertierenden Werken durchgeblättert. Mehrbändige Werke, bei denen mindestens ein Band im Zeitraum 1980-88 erschienen ist, werden komplett konvertiert. Das schon bei anderen Projekten Konvertierte wird überprüft, da in den Katalogen der Sonderstandorte die Mehrfachexemplare oft leider gar nicht oder nur unvollständig nachgewiesen sind.

Zahlreiche Kolleginnen und Hilfskräfte waren bisher unterschiedlich lange mit der Konversion befasst. Einige von innen wechselten inzwischen in andere Abteilungen oder Arbeitsbereiche.

Im Rahmen des Projektes Zukunftsoffensive Baden-Württemberg konnten drei zusätzliche Stellen besetzt werden.

Die mit der Konversion befassten Kolleginnen arbeiten in verschiedenen Räumen im Haus: In der Titelaufnahme, dem Zeitungslesesaal, der Landesbibliographie und in Räumen der früheren BfZ. Eine Liste, welche Katalogkästen gerade an welchem Standort bearbeitet werden, kann an der Auskunft eingesehen werden.

Mittlerweile sind wir beim Buchstaben F angelangt.

\section{Kunst- und Musiklesesaal}

Im Herbst 1999 wurde auf Wunsch der Direktion die Konversion des Haupt-AK zugunsten einer Konversion der Son- 
derstandortkataloge zurückgestellt, um in Zukunft die Titelkartenlieferungen für die Sonderstandorte einstellen zu können. Dies wird die Arbeit in allen betroffenen Arbeitsbereichen erleichtern.

Seit Anfang März ist auch der Kunstlesesaal konvertiert und der alphabetische Katalog des Musiklesesaals wurde in Angriff genommen. Dort sind zusätzlich zum Lesesaalbestand auch die im Magazin stehenden Werke zur Musik und die Noten im Musikmagazin verzeichnet; konvertiert wird aber nur der Bestand des Musiklesesaals.

\section{Handbibliothek Buchgeschichte}

Die Konversion des Kataloges der HB Buchgeschichte wird schätzungsweise im Sommer 2000 abgeschlossen sein.

\section{Ausblick}

Nur die Sonderstandorte Musiklesesaal, HB Auskunft und Karten-/GraphikLesesaal sind noch nicht konvertiert.
Nach Abschluss des Projektes am Alphabetischen Hauptkatalog wird lediglich der Magazinbestand der Jahre 1851 bis 1979 noch nicht maschinell erfasst sein.

Momentan (März 2000) sind für alle Bereiche der Konversion ca. 4 Stellen besetzt.

\section{Im SWB enthaltene Bestände}

- Die Bestände der Erscheinungsjahre 1500 - 1850 und 1989 ff.

- Die Bestände der Erscheinungsjahre 1980 bis 1988 bis zum Buchstaben $\mathrm{F}$ aus dem Alphabetischen PIKatalog

- Die Bestände des Hauptlesesaals, des Handschriften- und des Kunstlesesaals

- Über Bibeln folgt demnächst ein eigener Beitrag.

\section{Renate Wagner:}

\section{Bibliografien und Adressbücher zum Fach Musik jetzt im Musiklesesaal}

Im Sommer und Herbst 1999 wurde vollzogen, was schon über mehrere Jahre diskutiert und angedacht war, nämlich die bibliographischen Nachschlagewerke zum Fach Musik an einer Stelle zugänglich zu machen. Stand der Dinge war, dass einige Nachweismöglichkeiten im Musiklesesaal zu finden waren und andere in der HB Auskunft.

Die Verantwortlichen für die Arbeitsbereiche Musik bzw. Bibliographische Auskunft sind daher übereingekommen, die Teilung aufzuheben. Man entschied sich trotz geringerer Öffnungs- zeit des Musiklesesaals für die Umstellung aller Bibliographien dorthin. Vor allem ist nun eine fachlich spezifische und kompetente Auskunft an einer Stelle möglich.

Nachdem in der HB Auskunft durch o.g. Umstellung Platz gewonnen werden konnte, war es auch möglich, die Fachbibliographien zu verziehen. Vor allem bei den Sprachwissenschaften und der Geschichte konnten nun Engpässe entzerrt werden. Sie finden also bestimmte Bibliographien nicht mehr am gewohnten Platz. 